\title{
Review of In-Building Propagation Phenomena at UHF Frequencies*
}

\author{
Henry L. Bertoni, Seongcheol Kim" and Walter Honcharenko ${ }^{\#}$ \\ Center for Advanced Technology inTelecommunications \\ Polytechnic University \\ Brooklyn, NY 11201 \\ e-mail: hbertoni@prism.poly.edu
}

\begin{abstract}
When both the base station and subscriber antennas are located in the cluttered multipath environment inside buildings, fast fading is observed as either antenna is moved over a distance on the order of a wavelength. The fast fading is evident in measurements made on $C W$ signals, on individual arrivals for pulsed excitation, even for pulses as short as $5 \mathrm{~ns}$. The statistical properties of the fading are discussed, along with the usual measures of the pulse response, such as path loss, mean excess delay, rms delay spread and coherence bandwidth.
\end{abstract}

\section{Introduction}

Analysis of the radio communication channel characteristics is important for the development of wireless systems such as Personal Communication Systems (PCS), wireless Private Branch Exchanges (wPBX) and wireless Local area Networks (wLAN). Narrow band or CW path loss is a parameter that is used to define the spatial coverage of a base station, while the impulse response parameter such as mean excess delay and $\mathrm{rms}$ delay spread are useful in obtaining data rate and the bit error rate. The technical literature has many reports on $\mathrm{CW}$ path loss and the impulse response in the $800-900 \mathrm{MHz}$ band and in the 1.3, 1.4 and $1.8 \mathrm{GHz}$ bands [1-13]. Recently we have reported impulse response measurements made in the 2.4 GHz ISM band [20].

It is well known that a portable located in a cluttered environment receives signals arriving from many directions. For CW excitation, the interference between the different arrivals causes the received voltage amplitude to exhibit large spatial variations over distances that are on the order of a half wavelength. If the voltage is sampled at many closely spaced points over a distance of several wavelengths, the sampled values may be treated as a random distribution. When the receiver is obscured from the transmitter, no one signal arrival will be much stronger than many others, and the distribution of voltage amplitudes will approximate a Rayleigh distribution
[17] [18]. If a line of sight path exists between transmitter and receiver, the distribution will be Rician [17] [18]. For pulse excitation, some of the individually arriving signals will have relative delay that is less than the pulse width, and can therefore interfere. As a result, the amplitudes of the various pulses can also be expected to exhibit fast fading.

In this paper we examine the fast fading characteristics observed for CW and pulsed signals due to multiple arrivals for propagation inside buildings. When both the transmitter and receiver are located inside a building, the radio link is symmetrical and fast fading is observed when either end of the link is moved. We first show that averaging the received signal over position at one end of the link to remove the fast fading, as is common practice, does not eliminate the fading as the other end of the link is moved. In order to identify the path loss from one small area to another it is therefore necessary to average over both ends of the link. We subsequently consider the fast fading characteristics observed for pulsed signals of 5 ns duration. Even for such short pulses, identifiable pulses in the response are found to exhibit fast fading, indicating the presence of many propagation paths having differences in path length less than $1.5 \mathrm{~m}$

\section{Bi-Lateral Fading Characteristics}

The multipath components between transmitter and receiver inside a building are suggested by the ray paths in Figure 1, which is taken from [19]. The received signal will change as the location $x_{T}$ of the transmitter is moved a distance on the order of a wavelength because of the different phases imparted to the various rays. Conversely, if the transmitter is held fixed, and the receiver location $x_{R}$ is varied over a distance on the order of a wavelength, the phases of the arriving rays will also change, resulting in fading of the received signal. Depending on whether the location $x_{T}$ of the transmitter is at a maximum or a minimum of its fading pattern, the fading pattern as the receiver is moved will have a different average.

Measurements to confirm the foregoing speculation were made over one floor of a large office building $81 \mathrm{~m}$ by $50 \mathrm{~m}$

\footnotetext{
* This work was supported in part by Symbol Technologies, Inc., in part by a University Partnership in Research grant from Motorola, Inc., and in part by the New York State and Technology Foundation.

${ }^{\#}$ S.C. Kim and W. Honcharenko are now with AT\&T Bell Laboratories.
} 
In Building Propagation

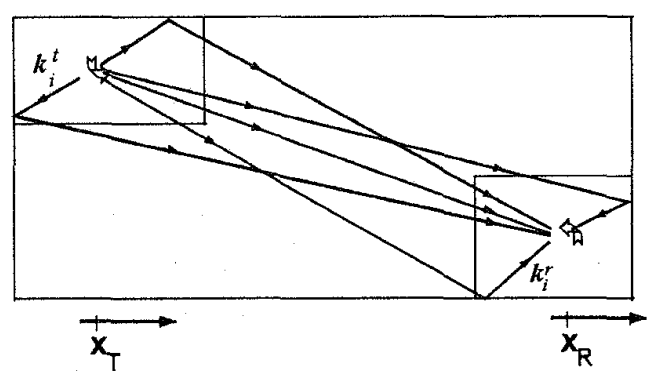

Transmitter in Clutter

Receiver in Clutter
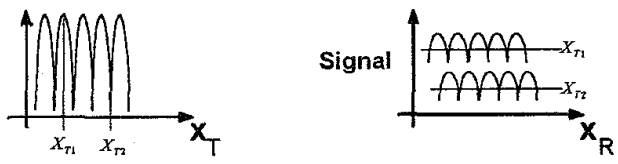

Fig. 1. Cartoon illustrating multipath environments at the transmitter and receiver, both of which are in the clutter.

using $825 \mathrm{MHz} \mathrm{CW}$ signals. The transmitter was a Motorola MX portable attached to a motor driven mechanism that moves the radio around a circle having a 3 foot $(0.91 \mathrm{~m})$ radius. This circle is in a plane parallel to and 6 feet $(1.82 \mathrm{~m})$ above the floor, and was traced out in 20.5 seconds.

The receiver system was located on a cart, to which a dipole antenna was fixed atop a pole. A small pointer attached to the bottom of the cart served as a location indicator, and the floor beneath the cart was marked off in 1 inch $(2.54 \mathrm{~cm})$ increments along a line having total length of 10 inches (25.4 $\mathrm{cm})$. The receiver sampling rate was 15 samples/sec, which allowed for approximately 300 samples per revolution of the transmitter. At a given receiver position, the mechanism was started from a due north orientation and samples of the received signal were taken. The receiver was then moved 1 inch from the previous location and the measurement process repeated. Prior to each measurement, the operators returned to chairs and remained motionless, although people were moving about elsewhere in the building.

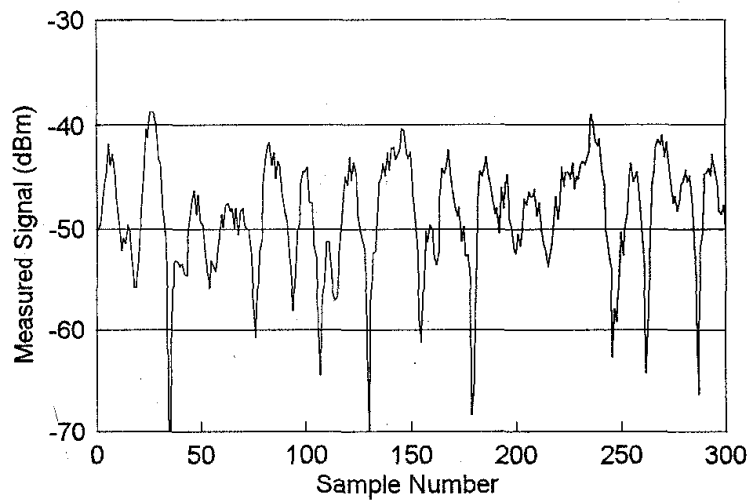

Fig. 2. Typical received signal for one revolution of robot arm as a function of sample number.
A typical plot of the received signal for one revolution of the transmitter around the circle is shown versus sample number in Figure 2 for one location of the receiving cart. Several fades or greater than $20 \mathrm{~dB}$ are evident in Figure 2. Because the circumference of the circle is $5.71 \mathrm{~m}$, or 15.7 wavelengths, 10 sample points were taken in a distance of approximately $1 / 2$ wavelength. It is seen in Figure 2 that many of the fades are separated by about 10 sample points, as is expected for the fading due to the interference of many ray arrivals.

The mean signal, rather than the average $\mathrm{dB}$, obtained for each receiver location was computed, and ranged from a high of $-42.2 \mathrm{dBm}$ to a low of $-46.5 \mathrm{dBm}$. The variation about the average of $44.9 \mathrm{dBm}$ is plotted in Figure 3 as a function of receiver location.

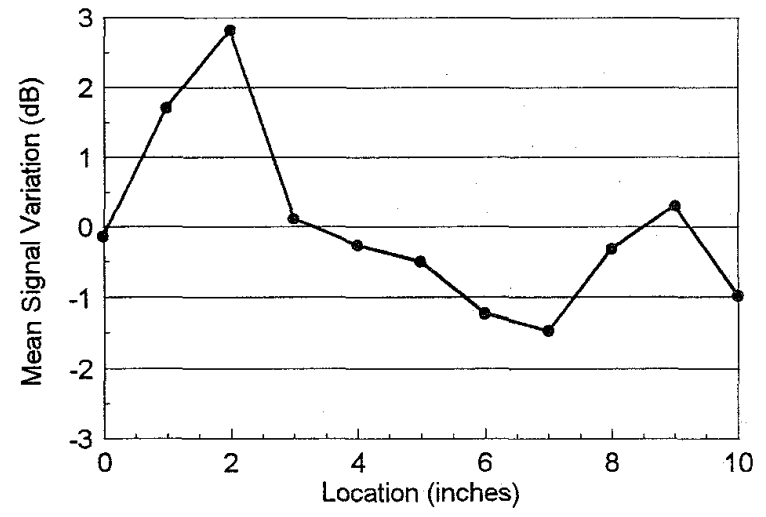

Fig. 3. Difference between the mean signals obtained for each measurement location and the bilateral sector average as a function of receiver location (inches).

It is seen that variation encompasses more than $4 \mathrm{~dB}$, implying that the true definition of the path loss from a small area to another requires averaging the received signal over the location of both ends of the radio link. This effect is not apparent in cellular applications, where one end of the link is located above the clutter, so that it is sufficient to average only over the position of the mobile. Alternatively, we can interpret the result as implying that using diversity at both ends of the link will improve performance more than using it only at one end.

\section{Impulse Response Measurements}

Channel sounding measurements were carried out in two different types of building using 5 ns pulses with a carrier frequency of $2.4 \mathrm{GHz}$. The first was a typical engineering building $60.5 \mathrm{~m}$ by $44 \mathrm{~m}$ consisting of a large open area divided into cubicles by head high movable partitions. This area is surrounded by rooms of different sizes, some of which are engineering laboratories. The partitions in the cubicle section are 1.57 meters high, while the acoustic drop ceiling was 2.9 meter above the floor. The transmitter was located at a cross isle between the cubicles near the center of the main cubicle area at a height of 2.5 meters. 
The second building was a large retail store having an open sales floor with storage and facilities rooms at the back of the building. The building is almost square with dimensions 100.5 $\mathrm{m}$ by $108.5 \mathrm{~m}$. Both interior and exterior walls are made of concrete blocks except for the $64 \mathrm{~m}$ long glass wall at the entrance to the building. The drop ceiling in the sales floor area is made of acoustic tiles and is about $4.27 \mathrm{~m}$ above the floor. There is no drop ceiling in the storage room. Two thirds of the sales floor has shelves supported of various heights ranging from 1.82 to $2.3 \mathrm{~m}$, and filled with a variety of metallic and nonmetallic sales items. The other parts of the sales floor have various kinds of metal racks and glass display cases whose heights are from 1 to 1.5 meters. Shelves between 1.82 and $2.44 \mathrm{~m}$ high are located in the storage room, and are filled with merchandise up to $3.35 \mathrm{~m}$. The transmitter was placed near the center of the building on the top of a merchandise shelf at a height of 2.95 meters.

A total of 10 receiver sites in the engineering building and 7 sites in the retail store were chosen for measurements. Four sites in the main cubicle area have a Line-of-Sight (LOS) path to the transmitter. For three sites located in the room or the corridor surrounding the main cubicle area, a single wall separated the receiver and transmitter, while at the remaining three sites they were separated by two or more walls. The receiver site located in the engineering laboratory is heavily cluttered by scatterers. In the retail store, four out of five sites on the sales floor had the path to the transmitter obstructed by the merchandise shelves. Two of these sites had light surrounding clutter whose height is comparable to that of the receiving antenna, and another two were heavily cluttered by the merchandise shelves.

At each measurement site, the receiver was located at 16 different positions separated by one quarter wavelength along a straight line. At each position the power delay profile $s(t)$ was recorded with the help of a portable computer for subsequent processing. For each type of receiver location (LOS, lightly cluttered, or heavily cluttered), similar results were obtained in both buildings.

\section{Overview of Measured Pulse Response}

Power delay profiles obtained from measured pulse responses can be separated into three different categories. The first category applies when a LOS path exists between the transmitter and the receiver; the second category consists of lightly obstructed paths; and the third category is for heavily obstructed paths. A series of typical power delay profile $s(t)$ for the 16 receiver locations at a LOS site and at a heavily cluttered site are shown in Figures 4(a), (b).

In Figure 4(a) it is seen that the power delay profile has a large initial pulse, followed by delayed pulses of small amplitude. Even though LOS conditions exist, the main received pulse is composed of two or more ray arrivals, as evidenced by the spatial fading as the receiver position is moved. Based on the heights of the two antennas and their separation, it appears that the fading is due to interference between the direct and floor reflected rays.

The power delay profiles in Figure 4(b) for a heavily cluttered site exhibit many individually identifiable peaks whose amplitudes vary rapidly with the location of the receiver.

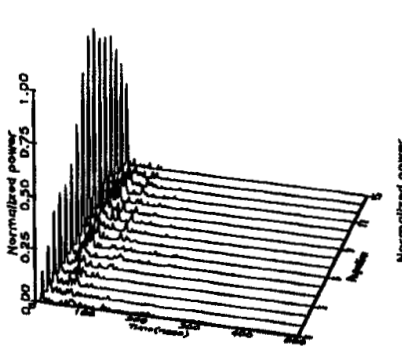

(a) $\operatorname{LOS}$

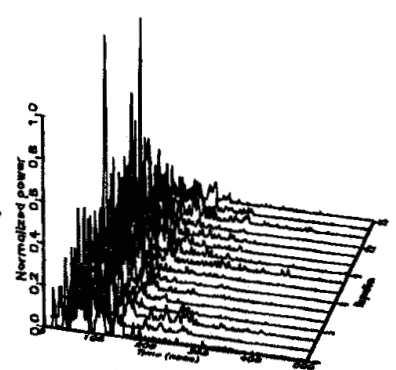

(b) Onstucted
Fig. 4. Individually arriving rays with small relative time delay cause fading of all fingers in the pulse response.
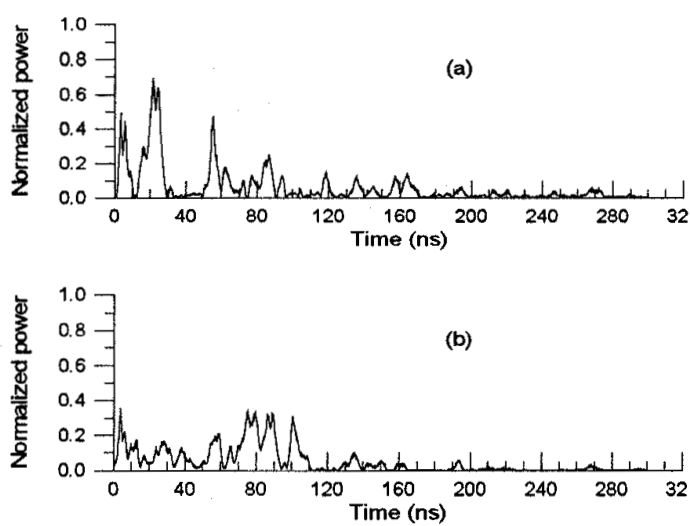

Fig. 5. Power delay profiles for sites with heavily obstructed paths to the transmitter in the engineering building.

This variation is shown more clearly in Figures 5(a), (b), where we have plotted the power delay profiles obtained at two positions separated by $12.3 \mathrm{~cm}$ along the radial direction from the transmitter. In these two figure there exist so many strong pulses that no one pulse dominates the response. The pulse near $20 \mathrm{~ns}$ in Figure 5(a) seems to be the strongest and overlaps with adjacent pulses. The amplitude of this pulse drops so much in Figure 5(b) that it is believed to be composed of many sub paths. This result can be explained by the postulate that phase differences between these sub paths change with movement of the receiver position, and as a result the amplitude of the superposition of the sub paths also changes. An examination of pulse responses at all of the 16 different receiver positions at this site reveals that none of the pulses have the same amplitude at all receiver positions. In addition to the amplitude variation of the strong pulses, the weak pulses appearing in late time of the response (out to 300 ns) also vary in amplitude as the receiver is moved. 


\section{Channel Characteristics}

Based on the power delay profile it is possible to determine statistical properties of the channel. Several such parameters are obtained below.

\section{5.a. Distribution functions of individual peaks}

The measured pulse response contains individual peaks that can be identified throughout the 16 individual receiver positions at each site, but are often jagged. In order to avoid a subjective choice of peak amplitude, and to accommodate all ray arrivals making up a peak we averaged the amplitudes over time bins, whose width is chosen as 6 ns so as to be slightly larger than the $5 \mathrm{~ns}$ pulse duration, centered at the delay time $T_{k}$ In this way, at each receiver position $\mathrm{m}$, we obtain for the time bin centered at $T_{k}$ an average pulse amplitude $A_{k, m}^{2}$ [10], [13]. The amplitude $A_{k, m}^{2}$ is then normalized to the average over the 16 receiver positions.

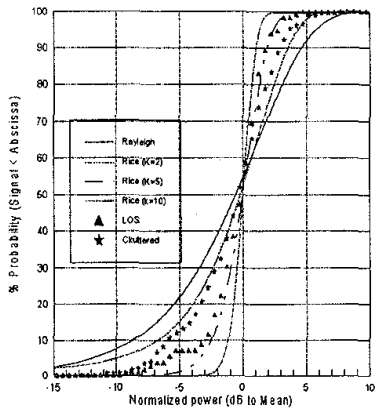

(a) Engineering building

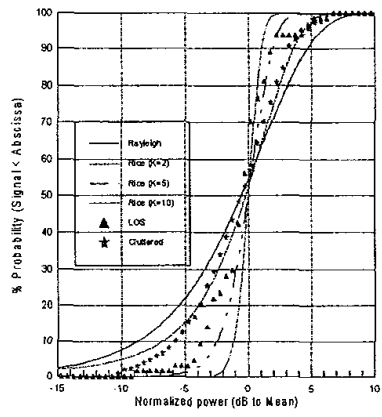

(b) Retail store
Fig. 6. Cumulative distributions of peak amplitudes

The normalized amplitudes of individual peaks were aggregated into separate data pools for LOS sites and cluttered sites in the two buildings. Figure $6(a),(b)$ represents the cumulative distribution functions of the data pools for $A_{k, m}$ corresponding to LOS and cluttered environments in the engineering building and the retail store, respectively. For LOS paths in both buildings, it is seen from Figure 6(a) and 6 (b) that the distribution of peak amplitudes are close to the Rician distribution curve with $K=5$, where $K$ is the ratio of the dominant signal to the standard deviation of other weaker and randomly varying signals [20]. At the cluttered sites, the measured distribution seems to follow the Rician curve with $K$ $=2$, which is very close to the Rayleigh distribution [17], [20]. The differences in the distributions for the LOS and for the cluttered environments is similar to that observed for $\mathrm{CW}$ excitation [12].

\section{5.b. Coherence bandwidth}

A digital communication system operating in the ISM band can use frequency hopping as a means for mitigating against fast fading. The coherence bandwidth is a measure of the minimum frequency difference such that the signals are not correlated with each other [14], [17]-[19]. If it is assumed that the impulse response represents a complex-valued wide-sense stationary zero mean Gaussian random process, then the frequency coherence of the channel can be found from the auto correlation function. the average power delay profile of the channel for pulse excitation $\langle s(t)\rangle$. Then the coherence bandwidth can be determined from the measured power delay profiles by taking the Fourier transform

$$
R_{H}(\Delta f)=\int_{-\infty}^{\infty}\langle s(t)\rangle e^{-j 2 \pi \Delta f t} d t
$$

The coherence bandwidth is then defined as the full width of $f$ at half the maximum of $R_{H}(f)$.

In Figures 7(a), (b) we have plotted the Fourier transform of the average power delay profile for typical LOS sites and heavily cluttered sites both in the engineering building. The site of the reviver for Figure 7(a) has a LOS path to the transmitter and has a power delay profile with only a few additional peaks after the first detectable peak, as in Figure 4(a). For this case it is seen that the coherence bandwidth is greater than $100 \mathrm{MHz}$. Figure $7(\mathrm{~b})$ is for a heavily cluttered site whose power delay profile contains many late time peaks, as in Figure 5. The coherence bandwidth at this site is approximately $7 \mathrm{MHz}$.

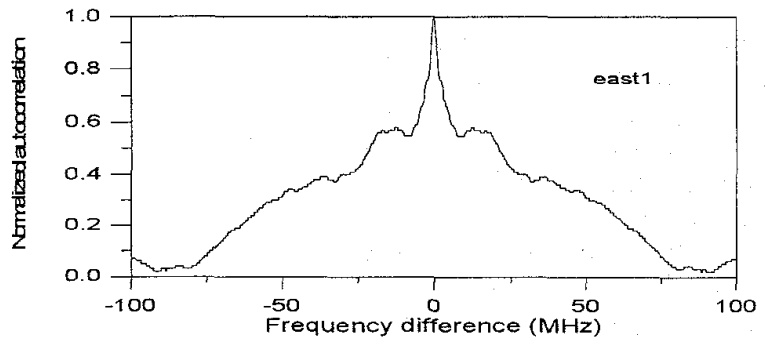

(a) LOS

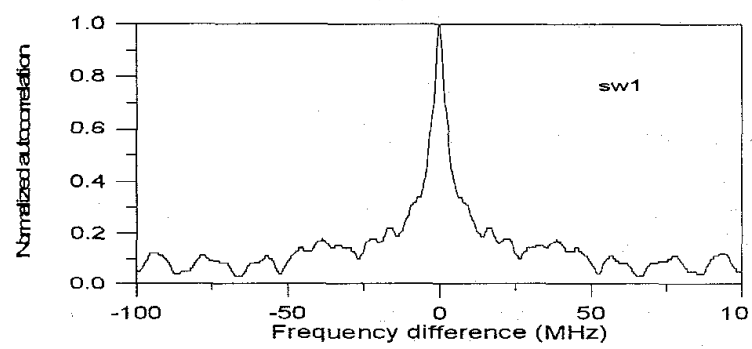

(b) Heavily cluttered

Fig. 7. Fourier transform of the power delay profiles at LOS and heavily cluttered measurement sites

\section{5.c. Mean excess delay and RMS delay spread}

In order to calculate the mean excess delay and the rms delay, the noise power was removed from the power delay profile $s(t)$ using the thresholding technique. Table 1 lists the range of values obtained for the mean excess delays and rms delay spread at all sites in the two buildings. For a sites with a LOS path, and even at most sites with lightly obstructed paths 
to the transmitter, the rms delay is larger than the mean excess delay by about $10 \mathrm{~ns}$. This results from the fact that the power delay profiles in these two environment are dominated by the first few strong pulses in the early time, which heavily influence the mean excess delay, while there are many weak pulses in the late time that influence the rms delay spread. However, at receiver sites having heavily obstructed paths, the rms delay spread and mean excess delay are nearly the same. For this case the power delay profiles are composed of many pulses arriving over about $200 \mathrm{~ns}$, so that pulses arriving long after the early pulses play an important role in determining both quantities [2], [5], [6], [10].

\begin{tabular}{|c|c|c|c|}
\hline & LOS & Partly Obstructed & Obstructed \\
\hline Mean Excess Delay & $16-31 \mathrm{~ns}$ & $31-83 \mathrm{~ns}$ & $61-117 \mathrm{~ns}$ \\
\hline RMS Delay Spread & $22-43 \mathrm{~ns}$ & $41-74 \mathrm{~ns}$ & $58-90 \mathrm{~ns}$ \\
\hline Coherence BW & $36-100 \mathrm{MHz}$ & $6-30 \mathrm{MHz}$ & $5-9 \mathrm{MHz}$ \\
\hline
\end{tabular}

Table 1. Pulse response characteristics

\section{Conclusions}

The indoor propagation environment generates a rich array of ray paths by connecting the transmitter and receiver. Because of the symmetry of the path when both transmitter and receiver are located in the environmental clutter, multipath interference effects occur at both ends of the radio link. It is necessary to provide diversity at both ends of the link if these effects are to be totally eliminated. Even for very short pulses, the multipath effect is evident in the various distinguishable pulse arrivals. Our measurements support the widely held belief that the amplitudes of these distinguishable arrivals are Rayleigh distributed for non-LOS links. The measurements of mean excess delay and rms Delay spread at $2.4 \mathrm{GHz}$ are similar to those obtained at lower frequencies.

\section{References}

[1] D. C. Cox "Delay doppler characteristics of multipath propagation at $910 \mathrm{MHz}$ in a suburban mobile radio environment," IEEE Transactions on Antennas and Propagation, vol. AP-20, No. 5, pp. 625-635, September 1972.

[2] D. M. J. Devasirvatham, "Time delay spread and signal level measurements of $850 \mathrm{MHz}$ radio waves in building environments," IEEE Transactions on Antennas and Propagations, vol. AP-34, No. 11, pp. 1300-1305, November 1986.

[3] S. J. Patsiokas, B. K. Johnson, and J. L. Dailing, "Propagation of radio signals inside buildings at 150,450 and 850 MHz," Proceedings of the $36^{\text {th }}$ IEEE Vehicular Technology Conference, Dallas, TX 1986, pp. 66-72.

[4] J. Horikosh, K. Tanaka, and T. Morinaga, "1.2 GHz band wave propagation measurements in concrete building for indoor radio communications," IEEE Transactions on Vehicular Technology, vol. VT-35, No. 4, pp. 146-152, November 1986.

[5] A. A. M. Saleh, and R. A. Valenzuela, "A statistical model for indoor multipath propagation," IEEE Joumal on
Selected Areas in Communications, vol. SAC-5, No. 2, pp. 138-146, February 1987

[6] D. M. J. Devasirvatham, "Multipath time delay spread in the digital portable radio environment," IEEE Communication Magazine, vol. 25, No. 6, pp. 13-21, June 1987.

[7] Y. Yamaguchi, T. Abe and T. Sekiguchi, "Radio propagation characteristics in underground streets crowded with pedestrians," IEEE Transactions on Electromagnetic Compatibility, vol. 30, No. 2, pp. 130-136, May 1988.

[8] H. W. Arnold and R. Murray, D. C. Cox, "815 MHz radio attenuation measured within two commercial buildings," IEEE Transactions on Antennas and Propagation, vol. 37, No. 10, pp. 1335-1339, October 1989.

[9] R. Bultitude, S. Mahmoud and W. Sullivan, "A comparison of indoor radio propagation characteristics at 910 $\mathrm{MHz}$ and $1.75 \mathrm{GHz}$, "IEEE Journal on Selected Areas in Communications, vol. 7, No. 1, pp. 20-30, January 1989.

[10] T. S. Rappaport, "Characterization of UHF multipath radio channel in factory buildings," IEEE Transactions on Antennas and Propagation, vol. 37, No. 8, pp. 1058-1069, August 1989.

[11] T. S. Rappaport, "Indoor radio communications for factories of the future," IEEE Communication Magazine, pp. 15-24, May 1989

[12] J. F. Lafortune, M. Lecours, "Measurement and modeling of propagation losses in a building at $900 \mathrm{MHz}$," IEEE Transactions on Vehicular Technology, vol. 39, No. 2, pp. 101-108, May 1990.

[13] G. L. Turin, F. D. Clapp, T. L. Johnston, S. B. Fine and D. Lavry, "A statistical model of urban multipath propagation," IEEE Transactions on Vehicular Technology, vol. VT-21, No. 1, pp. 1-9, February 1972.

[14] A. P. Bello and B. D. Nelin, "The effect of frequency selective fading on the binary error probability of incoherent and differently coherent matched filter receivers," IEEE Transactions on Communication System, vol. CS-11, pp. 170186, June 1963

[15] Simon Haykin, Communication Systems. New York: Wiley, 1983, Chapter 5.

[16] John G. Proakis, Digital Communications. New York: McGraw-Hill, 1989, pp. 702-719.

[17] William C. Y. Lee, Mobile Communications Engineering. New York: McGraw-Hill, 1982, Chapter 1, 2, 3, and 6.

[18] B. P. Lathi, Modern Digital and Analog Communication Systems. Philadelphia: Holt, Rinehart and Winston, 1989 , pp. 435-450.

[19] W. Honcharenko, H. L. Bertoni and J. L. Dailing, "Bilateral averaging over receiving and transmitting areas for accurate measurements of sector average signal strength inside buildings," IEEE Transactions on Antennas and Propagation, vol. 43, No. 5, pp. 508-512, May 1995.

[20] S. C. Kim, H. L. Bertoni and M. Stern, "Pulse propagation characteristics at $2.4 \mathrm{GHz}$ inside buildings," Accepted for publication in the IEEE Transactions on Vehicular Technology. 